\title{
Antibiogram of Bacterial Isolates from High Vaginal Swabs of Pregnant Women from Tertiary Care Hospital in Puducherry, India
}

\author{
N. Ravishankar ${ }^{1}$ and M. Prakash ${ }^{2} *$ \\ ${ }^{1}$ Research and Development Centre, Bharathiar University, Coimbatore-641 046, \\ Tamil Nadu, India \\ ${ }^{2}$ Department of Microbiology, Kanchi Shri Krishna College of Arts and Science, \\ Kilambi, Kancheepuram-631 551, Tamil Nadu, India \\ *Corresponding author:
}

\begin{tabular}{|l|}
\hline K e y w o r d s \\
Vaginal Discharge, \\
High Vaginal Swab, \\
Bacterial Vaginosis, \\
Vulvovaginal \\
Candidiasis. \\
\hline Article Info \\
\hline $\begin{array}{l}\text { Accepted: } \\
\text { 20 December } 2016 \\
\text { Available Online: } \\
\text { 10 January } 2017\end{array}$ \\
\hline
\end{tabular}

\section{A B S T R A C T}

Bacterial vaginosis is caused by an imbalance of the organisms that naturally exist in the vagina. The importance of bacterial vaginosis with respect to pregnant women's health is emphasized by the association between bacterial vaginosis and adverse outcome of pregnancy. The aim of present study was to evaluate the direct smear microscopy and culture for determination of bacteria from vaginal discharge of pregnant women. Vaginal discharge in women is sometimes caused by candida and aerobic bacteria organisms like E.coli, staphylococcus aureus, and $\beta$ - haemolytic streptococcus. Culture and sensitivity testing are done from high vaginal swab (HVS) specimen collected from women who come to the clinic complaining of vaginal discharge. Isolation and antibiotic sensitivity of these organisms are key to the successful treatment of the cause of vaginal discharge. This study tends to evaluate the microbial and antibiotic sensitivity and resistance pattern of high vaginal swab culture results in the Rajeev Gandhi government women and children hospital, Puducherry India. A total of 500 High Vaginal Swabs (HVS) were collected from pregnant women and were screened for bacterial population. For each patient one swab was used for smear preparation and Gram staining and the second swab was used for cultivation. HVS Samples culture and sensitivity results were reviewed. Out of which $252(50.4 \%)$ are the pathogenic organisms and 248(49.6\%) yielded growth of non pathogenic organisms. Candida species were the predominant microbial organism, $27 \%$ (68/252). Bacterial Vaginosis causing bacteria identified were mostly the aerobic types (184); with Escherichia coli being predominant, 38.8\% (98/184) followed by Klebsiella sps, and Staph. aureus. Pregnant women of age group 20-30 are showing more pathogenic isolates growth. Amikacin (89.6\%), Norfloxacin (75.6\%), Ciprofloxacin (66.2\%) and Gentamycin $(68.4 \%)$ revealed high levels of susceptibility. Whereas high resistance rates were observed for Amoxicillin (83.3\%) Tetracyclin (65.4\%) and Cotrimoxizole (62.7\%). The cephalosporins showed the best antibiotic sensitivity. Candida spp. and aerobic bacteria were the predominant microbial organisms identified from HVS results in the Puducherry. Proper laboratory diagnosis to identify causative organisms is vital for optimal therapeutic outcome. 


\section{Introduction}

The vaginal microflora constitutes a complicated environment, composed of varying microbiological species in variable quantities and proportions and their concentrations are indicative of the vaginal health of the individual (Donders et al., 2005). The microbial ecology subject to remarkable changes over the course of lifetime a, induced by developmental and hormonal changes (Pybus et al., 1999). In childhood, the vaginal flora contains skin commensals and bowel organisms. At menarche, the $\mathrm{pH}$ falls from neutral to approximately 4, and the flora becomes dominated by lactobacilli. Many other organisms may be present in lower concentrations, including anaerobic and facultative anaerobic bacteria and Candida spp. (Donders, 1999). In women of childbearing age this system is also dominated by Lactobacillus spp., a defining characteristic of which is the ability to grow in acid media and tolerate acid conditions at $\mathrm{pH}$ around 4.5; lactobacilli also ferment carbohydrates to produce lactic acid (Sobel, 2000). The normal vaginal bacterial flora of healthy premenopausal women continues to consist predominantly of Lactobacillus spp. These are believed to play a protective role in guarding the urogenital tract against infection by pathogens (Romanik and Martirosian, 2004). Bacterial vaginosis (BV) is caused by an imbalance of the organisms (flora) that naturally exist in the vagina. BV is among the diseases that most frequently associated with vaginitis. The other diseases are vulvovaginal candidiasis, and trichomoniasis. Vaginitis is usually characterized by vaginal discharge, vulvar itching and irritation, or odor (Witt et al., 2002).

Normally, about $95 \%$ of vaginal flora is lactobacillus bacteria. These lactobacilli help keep the vaginal $\mathrm{pH}$ level low and prevent overgrowth of other types of organisms. In bacterial $\mathrm{BV}$, a condition characterized by a raised vaginal $\mathrm{pH}$ and milky discharge, the normal vaginal flora is replaced by a mixed flora of aerobic, anaerobic and microaerophilic species (Schwebke, 2000). It seems that $\mathrm{BV}$ is accompanied by a shift in the normal Lactobacillus flora to a mixed vaginal anaerobic flora including Gardnerella vaginalis, Bacteroides spp., and Mobiluncus spp. (Wilks et al., 2004). BV commonly occurs in women of childbearing age and can be especially substantial in pregnant women (Witt et al., 2002). Prevalence of 10 to $31 \%$ has been reported in various populations (Wolrath et al., 2001; Chaudry et al., 2004). Women with BV have fewer Lactobacillus organisms than normal and more of other types of bacteria (Koumans et al., 2002). The importance of BV with respect to women's health is emphasized by the association between BV and pelvic inflammatory diseases, adverse outcome of pregnancy, postpartum endometritis, and cuff cellulites (Donders et al., 2005; Berg, 2001). Women with BV during pregnancy, have higher risks of miscarriage, early (preterm) delivery, and uterine infection after pregnancy (Leitich et al., 2003). Centers for disease control and prevention (CDC) advise that all pregnant women with BV symptoms be screened and treated with antibiotics (CDC, 2002).

\section{Materials and Methods}

The study population was comprised of 500 pregnant women with gestation age ranging between 18 to 40 years referred to Obstetrics and Gynecology department of Rajiv Gandhi Government women and Child hospital, Pondicherry from January 2014 to June 2016.

\section{Specimen Collection and Processing}

Sampling was carried out by insertion of two sterile cotton swabs for diagnosis of flora and $\mathrm{BV}$, one swab was obtained for smear preparation and Gram stain and the other 
swab was obtained for culture. Samples were immediately transferred to the microbiology laboratory. Gram stained smears were examined under oil immersion (x 1,000) of light microscope for the following morphotypes: large Gram positive rods (Lactobacillus morphotypes), small Gram variable and Gram- negative rods (Gardnerella and Bacteroides morphotypes), and curved Gram variable rods (Mobilincus morphotypes).

\section{Culture and Sensitivity}

High vaginal swabs specimen were received from patients seen within the hospital either on admission or from gynecology outpatient department. In either case, specimens were collected by trained nurses and Doctor's. Patients demographics were collected, and few drops of normal saline added to the collected specimen for microbial examination. Specimens were then inoculated into MacConkey agar, blood agar and/or chocolate agar, prepared from nutrient agar (Tulip Diagnostic Limited, India) for investigation of BV and normal flora. Standard culture and sensitivity procedures were followed and the results interpreted. The culture plates were incubated in $37^{\circ} \mathrm{C}$ in presence of $5 \% \mathrm{CO} 2$ for 24-48 h. The culture plates were then examined and based on grown bacteria, the necessary biochemical tests were performed and the organisms were identified as per standard criteria (Forbes et al., 2007). Antibiotic susceptibility was tested using disc diffusion method (modified Kirby-Bauer's method). Drugs used for the susceptibility patterns: amikacin-AK, amoxyclav-AMC, azithromycin-AZM, chlorampinicol-C, ceftazidime-CAZ, ceforoxime-CXM, cephotaxime-CTX, ceftriaxone-CTR, ciprofloxacinCIP, co-trimoxazole-COT, cepoperazone + sulbactam-CFS, doxycycline-DO, gentamicin-G, norfloxacin-NA, ofloxacin-OF, levoflox-LE, oxacillin-OX, meropenem-MR, pipercillin/tazobactam-PT, vancomycin-VA.

\section{Results and Discussion}

Total High Vaginal Sample (HVS): 500

Total Positives: 252 (50.4\%)

Out of 500 samples enrolled in the study, 252 $(50.4 \%)$ had positive vaginal cultures. Fifteen microorganisms, including non pathogenic bacteria like Micrococcai, Diphtheroid and Lactobacillus spp., and pathogenic gramnegative bacteria, gram positive coccai, candida spp., were isolated. The highest infection rate was observed among women aged between 20 and 30 years $(61.0 \%)$, followed by those aged 31-40 years $(27.0 \%)$ and more than 40 years $(6.5 .0 \%)$, and the lowest frequency of infection was observed among those aged between 18 and 20 years $(5.5 \%)$, as shown in Figure.1.

According to Table 1. Incidence of E. coli is the highest among all the cases i.e. $38.88 \%$. Then comes the Candidial species amounting $26.98 \%$ followed by Klebsiella sps $13.88 \%$, and Staphylococci 10\%. At the lowest level come the Enterococcus $1.58 \%$, Acinetobacter, Streptococcus, Pseudomonas are 5\% each, Coagulase Negative Staphylococcus (CoNS) and Proteus spps are $0.79 \%$ each, Providencia is $0.39 \%$. Among the 252 isolates 146 $(57.93 \%)$ are the gram negative bacteria, 38 $(15.07 \%)$ are the gram positive bacteria and $68(26.98 \%)$ are the candida isolates. Gram Negative bacteria are mostly resistant to penicillins (amocyclav/amoxicillin), Fluroquinoles (ciprofloxacin, levofloxacin, ofloxacin ). The antibiotic sensitive pattern of E.coli showed a higher sensitivity towards piperacillin/tazobactam (85\%), cefoperazone/ sulbactum (88) and amikacin (91\%). The antibiotic sensitivity pattern of Klebsiella showed a higher sensitivity towards cefoperazone/ sulbactum (89\%), amikacin $(85 \%)$. E.coli strains were more resistant to Amoxycillin $85 \%$, cefuroxime $89 \%$, ceftriaxone 69\%, ciprofloxacin 63\%, 
cotrimaxole 54\%. Klebsiella showed comparatively less resistant to ciprofloxacin $51 \%$, cotrimaxole $55 \%$, cefuroxime $69 \%$, and it was highly resistant to Amoxicillin 90\%. Gram-positive organisms tested against vancomycin and oxacillin, resistance was found. In conclusion, E coli was found to be the common cause of UTI among the pregnant women. Low to moderately high level of resistance was found in first line drugs while high level of resistance was found in third generation cephalosporin. It is recommended to monitor the levels of resistance for nitrofurantoin, fluoroquinolone (ciprofloaxacin, norfloxacin) and cefotaxime. Over the past years the susceptibility to cephalosporins tends to decrease. Amyloglycosides, piperacillin-tazobactam, amikacin and cefoperazone/sulbactum show high antibacterial activity as shown.

The majority of pregnant women were in age group of 21-30 years and gestational ages. represent the relative frequencies of the most common isolated normal flora and a few pathogenic bacteria in stained smears and culture. The majority of pathogenic isolated bacteria in culture were Escherichia coli, Klebsiella, yeast, and Staphylococci. Enteric Gram-negative rods and Streptococci were isolated at lower rate. Based on the results of Gram-stained smears, 78(65\%) Gram positive rods and 54 (45\%) Gram positive cocci were detected. Gram-stained smears were showed to include polymorphonuclear leukocytes, fungi and clue cells.

Vaginal infections have wide implications for women's health, being the most common gynaecological problem. It is believed that the lactobacilli play an important role in maintaining normal vaginal ecosystem and preventing the growth of opportunistic bacteria. The present study demonstrates the prevalence of potential vaginal pathogens in symptomatic pregnant women. The results of present study are comparable to the study by
Lakshmi et al., which compared the prevalence of vaginal infections between premenopausal and postmenopausal women. Increased infections in post-menopausal women are due to the vagina being colonized by pathogenic organisms more than the protective organisms. The highest frequency of infection was noted at an age of 20 to 30 years with a fall in the frequency of infection as age advanced. Similar results with respect to age were seen in a Kenyan study, although it was limited to the study of only one organism.

Several microorganisms were isolated in present study, and those with the highest frequencies were Escherichia coli, Klebsiella sps, Staph aureus, Streptococcus, Pseudomonas aeruginosa, Proteus sp., Micrococci, Enterococci, Acinetobacter, and Candida albicans. Commensal growths were found in $25 \%$ of cases that need not be treated, but necessary measures such as identification of risk factors (douching, sprays, diabetes) and their prevention have to be carried out.

The study also found Escherichia coli to be the most common pathogenic bacteria $(38.8 \%)$ isolated from culture. In fact, Escherichia coli was found to be the most prevalent pathogen isolated not only from high vaginal swabs but also urine, pus, blood and wounds, as seen in a study conducted by Dutta et al., in Dhaka. Candida albicans is tolerant to the acidic environment and is hence found in the vagina, but the concentrations are too low to cause symptoms. In conditions of decreased local immunity, the hyphae would multiply and transform into infective patterns that result in symptomatic vaginitis.18 Colonization of Candida species also happens during pregnancy, resulting in symptomatic vaginitis.19. Infections with MRSA became a global health issue in 1960s, when the strains were first identified. 
Table.1 Distribution of organisms from HVS samples total with \%

\begin{tabular}{|c|l|c|c|}
\hline S.No. & Isolated Organism & $\begin{array}{l}\text { No. of Positive } \\
\text { isolates from HVS }\end{array}$ & $\begin{array}{c}\text { Positive isolates } \\
\text { from HVS (\%) }\end{array}$ \\
\hline 1 & E.coli & 98 & 38.88 \\
\hline 2 & Klebsiella species & 35 & 13.88 \\
\hline 3 & Staphylococcus aureus & 27 & 10.71 \\
\hline 4 & Enterococcus & 4 & 1.58 \\
\hline 5 & Acinitobacter & 5 & 1.98 \\
\hline 6 & CoNS & 2 & 0.79 \\
\hline 7 & Streptococcus & 5 & 1.98 \\
\hline 8 & Pseudomonas species & 5 & 1.98 \\
\hline 9 & Proteus species & 2 & 0.79 \\
\hline 10 & Providenciae & 1 & 0.39 \\
\hline 11 & Candida & $252(50.4)$ & 26.98 \\
\hline & & & 99.94 \\
\hline
\end{tabular}

Table.2 Bacterial Isolates from HVS samples Resistance patterns to the antibiotics

\begin{tabular}{|l|c|c|c|c|c|c|c|c|c|c|}
\hline Isolates & $\begin{array}{c}\beta- \\
\text { Lactum }\end{array}$ & \multicolumn{3}{|c|}{ Cepholosporin } & $\begin{array}{c}\text { Tetracy- } \\
\text { cline }\end{array}$ & $\begin{array}{c}\text { Carbape } \\
\text {-nems }\end{array}$ & \multicolumn{2}{c|}{ Amyloglycosides } & \multicolumn{2}{l|}{ Quinolones } \\
\hline & AMC & CXM & CTX & CTR & DO & MR & G & AK & LE & OF \\
\hline E. coli & 88 & 55 & 46 & 57 & 43 & 19 & 32 & 2 & 17 & 11 \\
\hline Klebsiella & 29 & 25 & 25 & 28 & 15 & 12 & 11 & 4 & 16 & 17 \\
\hline Staphylococcus aureus & 2 & 6 & 4 & 6 & 5 & 7 & 8 & 1 & 5 & 7 \\
\hline Enterococcus & 2 & 2 & 1 & 2 & 1 & 1 & 0 & 0 & 1 & 2 \\
\hline Acinitobacter & 1 & 4 & 2 & 4 & 2 & 2 & 1 & 0 & 3 & 2 \\
\hline CONS & 1 & 1 & 1 & 2 & 0 & 0 & 0 & 1 & 1 & 0 \\
\hline Pseudomonas species & 2 & 2 & 1 & 0 & 1 & 2 & 1 & 0 & 1 & 1 \\
\hline Proteus species & 1 & 0 & 1 & 0 & - & - & 1 & 0 & 2 & 1 \\
\hline Providenciae & 2 & 1 & 1 & 1 & 2 & 3 & - & 0 & 1 & - \\
\hline
\end{tabular}

AMC-Amoxicillin, CXM-Ceforoxime, CTX-Cephotaxime, CTR-Ceftriaxone, DO-Doxycycline, G-Gentamicin,

DO-Doxycyclin, MR-Meropenem, AK-Amikacin, NX-Norfloxacin, LE-Levofloxacin, OF-Oflxacin

Table.3 No. of MDR strains isolated from HVS samples of pregnant women

\begin{tabular}{|l|c|}
\hline Isolates & HVS samples \\
\hline E.coli & 60 \\
\hline Klebsiella & 21 \\
\hline S. aureus & 2 \\
\hline Enterococcus & 1 \\
\hline Acinetobacter & 3 \\
\hline CONS & 1 \\
\hline Pseudomonas & 2 \\
\hline Proteus & 2 \\
\hline Streptococcus & 1 \\
\hline
\end{tabular}


Fig.1 Prevalence of Bacterial infection in pregnant women Age group

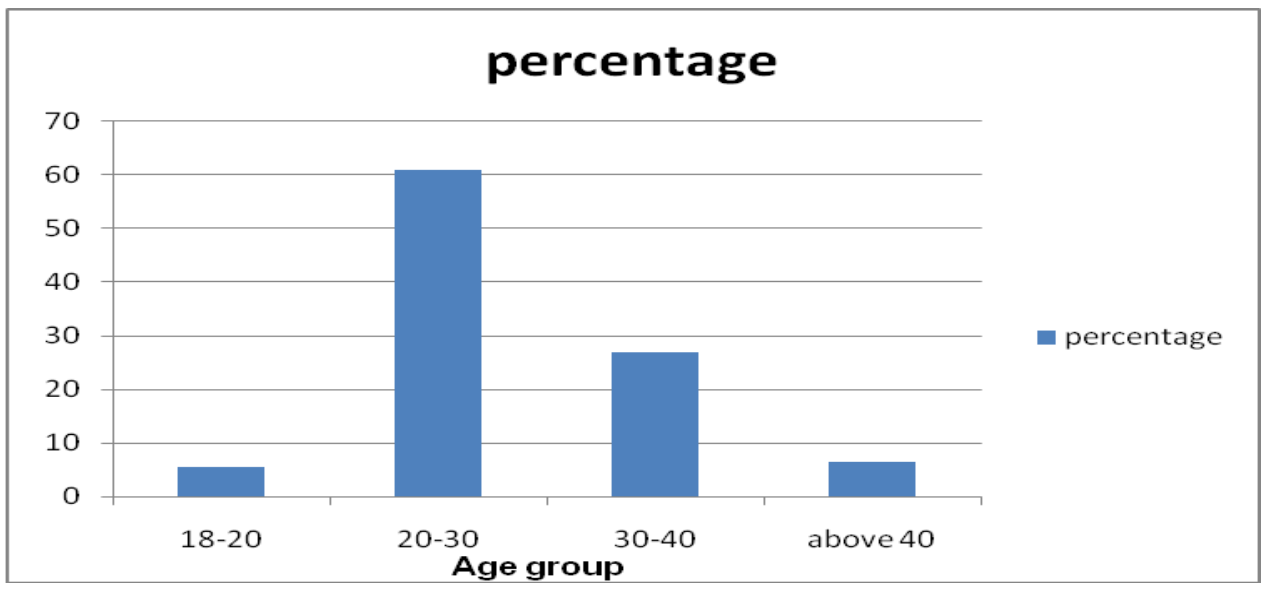

Fig.2 Distribution of Organisms isolated from HVS samples

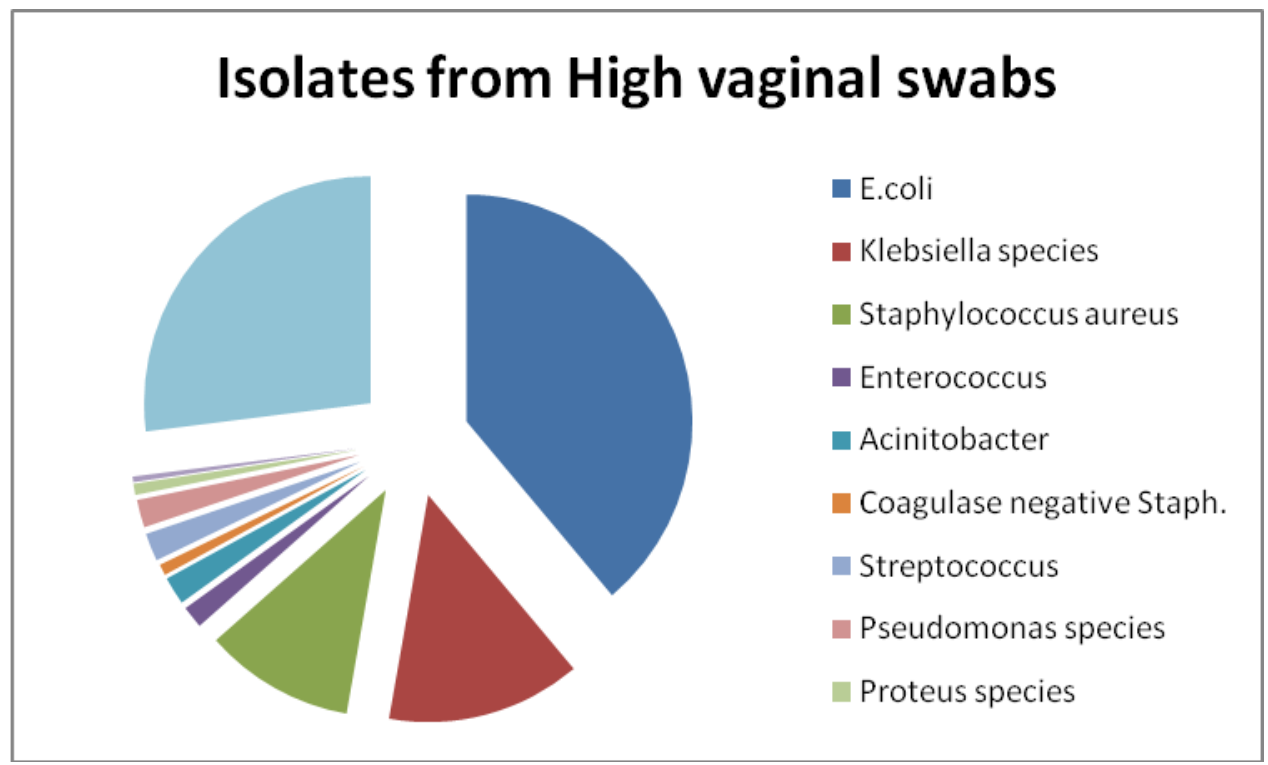

They may be acquired nosocomially or from the community. What make them difficult to treat are their multiple antibiotic resistant profiles and wide varying prevalence. One should keep in mind that higher antibiotics may be required to treat these infections.

Maternal group B Streptococci (GBS) colonization is a major risk factor for GBS disease in neonates. In pregnant women, GBS causes cystitis, amnionitis, endometritis and stillbirth; occasionally, it leads to endocarditis or meningitis.
Coagulase-negative Staphylococci (CoNS), which are considered to be skin commensals, were found in $0.79 \%$ of the cases. No cases of trichomoniasis, Chlamydia infection, and Neisseria gonorrhoeae infection were noted.

The presence of co-morbidities like hospitalization, immunosuppression and coexistent reproductive tract infections have to be evaluated accordingly. It is known that vaginal infections, due to a disruption of normal vaginal flora, increase the risk of sexually transmitted infection, especially 
human immunodeficiency virus (HIV). However, our study did not identify any association with HIV, and diabetic status records were not available. No patients had taken hormone supplements or any other medications that could interfere with the results.

Our study has several limitations. The practice of swab culture is done mostly in clinical microbiological laboratories, and clinical diagnosis may be suboptimal. Only regularly used antibiotics were included in the sensitivity testing, and socio-demographic factors have not been considered. These limitations have to be overcome by future studies, and proper practices have to be implemented in order to preserve these lifesaving drugs for the future.

Diagnosis of these infections based on culture sensitivity is a definite step in treatment of these infections. In regular practice, fixed protocols are followed. Inadequate treatment with antimicrobials due to non-compliance or under the prescription of drugs results in high incidence of recurrence. Extensive resistance rates have emerged among commonly used antibiotics due to indiscriminate use. Newer antibiotics like imipenem and meropenem are highly effective but expensive.

A significant proportion of pathogens causing vaginal infections are resistant to the conventionally used antibiotics. This study is a step in familiarizing sensitivity and resistance patterns to used antibiotics, preventing resistance and thus preventing the chronic sequelae. Thus, the present study raise a question of changing the syndromic protocol to treatment protocol based on culture sensitivity. Substantial health gains with a reduction of the disease burden among women should be the long term goal of treatment which should be intended with knowledge of culture sensitivity.
In conclusion, vaginal discharge in pregnancy is common, but distinguishing abnormal vaginal discharge from normal leucorrhoea of pregnancy is challenging. Bacterial vaginosis, candidiasis are a common problem in women reproductive age. Since findings have showed that the trio of vaginal candidiasis, trichomoniasis and bacterial vaginosis are common causes of abnormal vaginal discharge in pregnancy; efforts must be made to exclude these conditions in pregnant patients presenting with vaginal discharge so that appropriate treatment can be instituted timely. Moreover ciprofloxacin, norfloxacin, and gentamycin are the recommended drugs for empiric therapy and prophylaxis as needed.

\section{References}

Ahmed HM, Rasool VH, Al-Tawil NG. Prevalence of abnormal vaginal signs and symptoms among attendees of Obstetrics and Gynecology Department of Shaqlawa Hospital. Medical Journal of Babylon. 2014;11(1):120-9.

Amsel R, Totten PA, Spiegel CA, Chen KCS, Eschenbach DA, Holmes KK. Nonspecific vaginitis: diagnostic criteria and microbial and epidemiologic associations. Am J Med 1983; 74: 14-22.

Aubyn GB, Tagoe DN. Prevalence of vaginal infections and associated lifestyles of students in the University of Cape Coast, Ghana. Asian Pac J Trop Dis. 2013 Aug;3(4):267-70.

Barlette JJ, Berg AO, Heidrich FE (1999). Clinical comparism of microscopic and culture techniques in the diagnosis of vaginal infections. J. Fam. Prac., 2(3): 24-33.

Bergström S. Infection-related morbidities in the mother, fetus and neona- te. J Nutr. 2003 May;133(5 Suppl 2):1656S$1660 \mathrm{~S}$. 
Burrow RC, Bueshing UJ (1999). Bacterial vaginosis in virgins and sexually active females: Evidence against exclusive sexual transmission. J. Obstet. Gynecol., 8(5): 97 -99.

Cheesbrough M (2000). Microbiological test in; District laboratory practice for tropical countries part 2, Cambridge Low Price Edition, Cambridge University Press; pp. 69-71.

Cruickshank DR, Sharman PO (1994). Epidemiology and outcomes associated with moderate to heavy Candida colonization. Am. J. Intern. Med., 149(78): 33-35. Dodson MG, Friedrich EG (1997). Psychosomatic vulvovaginitis. Obstet. Gynecol., 51(23): 98.

Eschenbach DA, Hillier S, Critchoer C (1999). Diagnosis and Clinical manifestation of bacterial vaginosis. $\mathrm{J}$. Obstet. Gynecol., 16 (8):

Fisher B (1993). The Epidemiology of vulvovaginal candidiasis, risk factors AMF Public Health., 80: 329-331.

Germain M, Krohn MA, Hillier SL, Eschenbach DA. Genital flora in pregnancy and its association with intrauterine growth retardation. J Clin Microbiol 1994; 32: 2 162-2 168.

Gravett MG, Nelson HP, DeRouen T, Critchlow C, Eschenbach DA, Holmes KK. Independent associations of bacterial vaginosis and Chlamydia trachomatis infection with adverse pregnancy outcome. J Am Med Assoc 1986; 256: 1899-1903.

Hay PE, Lamont RF, Taylor-Robinson D, Morgan DJ, Ison C, Pearson J. Abnormal bacterial colonisation of the genital tract and subsequent preterm delivery and late miscarriage. BMJ

Hill GB, Eschenbach DA, Holmes KK. Bacteriology of the vagina. Scand $\mathrm{J}$ Urol NephroI 1985; Suppl 86: 23-39.

Hillier SL, Krohn MA, Nugent Rp, Gibbs RS for the Vaginal Infections and Prematurity Study Group. Characteristics of three vaginal flora patterns assessed by Gram stain among pregnant women. Am J Obstet Gynecol 1992; 166: 938-944.

Holst E, Rossel Goffeng A, Andersch B. Bacterial vaginosis and vaginal microorganisms in idiopathic premature labor and association with pregnancy outcome. J Clin Microbiol 1994; 32: 176-186.

Jejeebhoy S, Koenig M, Elias C, editors. Investigating reproductive tract infections and other gynaecological disorders: a multidisciplinary research approach. Cambridge (UK): Cambridge University Press; 2003. p. 30-81.

Karou SD, Djigma F, Sagna T, Nadembega C, Zeba M, Kabre A, et al. Antimi- crobial resistance of abnormal vaginal discharges microorganisms in Ouagadougou, Burkina Faso. Asian Pac J Trop Biomed. 2012 Apr;2(4):294-7.

Lamont RF, Taylor-Robinson D, Newman M, Wigglesworth J, Elder MG. Spontaneous early preterm labour associated with abnormal genital bacterial colonization. $\mathrm{Br} \mathrm{J}$ Obstet Gynaecol 1986; 93: 804-810.

Lamont RF, Taylor-Robinson D, Wigglesworth JS, Furr PM, Evans RT, Elder MG. The role of mycoplasmas, ureaplasmas and chlamydiae in the genital tract of women presenting in spontaneous early preterm labour. J Med Microbiol 1987; 24:

Lamont RF. Bacterial vaginosis. In: Studd J (ed) 1994 Yearbook of the Royal College of Obstetricians \& Gynaecologists. London: Parthenon Publishing Group. 1995; 149-1 60.

Larsen B, Monif GR. Understanding the bacterial flora of the female genital tract. Clin Infect Dis. 2001 Feb 15;32(4):e69-77. 
Lehman R. Clinical approach to recognizing and managing a patient with vaginal atrophy: a guide for physician assistants. Internet Journal of Academic Physician Assistants. 2009;8(1).

McDonald HM, O'Loughlin JA, Jolley P, Vigneswaran R, McDonald PJ. Vaginal infection and preterm labour. $\mathrm{Br} \mathrm{J}$ Obstet Gynaecol 1991; 98: 427435.

Mead PB. Epidemiology of bacterial vaginosis. Am J Obstet Gynecol 1993; 169: 44H49. 4. Eschenbach DA, Hillier S, Critchlow C, Stevens C, DeRouen T, Holmes KK. Diagnosis and clinical manifestations of bacterial vaginosis. Am J Obstet Gynecol 1988; 158: 819828.

Nugent RP, Krohn MA, Hillier SL. Reliability of diagnosing bacterial vaginosis is improved by a standardized method of gram stain interpretation. J Clin Microbiof 1991; 29: 297-301.

Puri R, Malhotra J. Recurrent urinary tract infection (UTI) in women. South Asian Federation of Obstetrics and Gynecology. 2009 Jan-Apr;1(1):10-3.

Ravel J, Gajer P, Abdo Z, Schneider GM, Koenig SS, McCulle SL, et al. Vagi- nal microbiome of reproductive-age women. Proc Natl Acad Sci U S A. 2011 Mar;108(Suppl 1):4680-7.

Reid G, Bruce AW, McGroarty JA, Cheng $\mathrm{KJ}$, Costerton JW. Is there a role for lactobacilli in prevention of urogenital and intestinal infections? Clin Microbiol Rev 1990; 3: 335-344.

Romero R, Hassan SS, Gajer P, Tarca AL, Fadrosh DW, Nikita L, et al. The composition and stability of the vaginal microbiota of normal pregnant wo- men is different from that of non-pregnant women. Microbiome. 2014 Feb $3 ; 2(1): 4$.

Soper DE, Bump RC, Hurt WG. Bacterial vaginosis and trichomoniasis vaginitis are risk factors for cuff cellulitis after abdominal hysterectomy. Am J Obstet Gynecol 1990; 163:

Spiegel CA, Amsel R, Holmes KK. Diagnosis of bacterial vaginosis by direct Gram stain of vaginal fluid. J Clin Microbiol 1983; 18: 170-177.

Watts DH, Krohn MA, Hillier SL, Eschenbach DA. Bacterial vaginosis as a risk factor for post-cesarean endometritis. Obstet Gynaecol 1990; 75: $52-58$.

\section{How to cite this article:}

Ravishankar, N., and Prakash, M. 2017. Antibiogram of Bacterial Isolates from High Vaginal Swabs of Pregnant Women from Tertiary Care Hospital in Puducherry, India. Int.J.Curr.Microbiol.App.Sci. 6(1): 964-972. doi: http://dx.doi.org/10.20546/ijcmas.2017.601.114 